\title{
Health-related quality of life, fatigue, and posttraumatic growth of cancer patients undergoing radiation therapy: A longitudinal study
}

Zsuzsanna Tanyi ${ }^{a}$, Kornélia Szluha ${ }^{\text {, }}$ László Nemes ${ }^{\text {a }}$, Sándor Kovács ${ }^{c}$, Antal Bugán $^{\mathrm{a}}$

${ }^{a}$ Department of Behavioural Sciences, Medical and Health Science Center, University of Debrecen, Debrecen, Hungary; ${ }^{b}$ Department of Radiotherapy, Medical and Health Science Center, University of Debrecen, Debrecen, Hungary; ${ }^{c}$ Department of Economic Analysis and Statistics, Institute of Economic Analytical Methodology and Applied Informatics, University of Debrecen, Debrecen, Hungary

Corresponding author: Zsuzsanna Tanyi

Department of Behavioural Sciences, Medical and Health Science Center, University of Debrecen

Hungary, H-4032 Debrecen, Nagyerdei krt. 98.

Email: tanyi.zsuzsanna@gmail.com

Phone: +36-52- 411-600/56230

Fax: +36 (52) 255-487

Kornélia Szluha

Department of Radiotherapy, Medical and Health Science Center, University of Debrecen Hungary, H-4032 Debrecen, Nagyerdei krt. 98.

Email: szluha.kornelia@gmail.com

Phone: +36-52- 453-585 
László Nemes

Department of Behavioural Sciences, Medical and Health Science Center, University of Debrecen

Hungary, H-4032, Nagyerdei krt. 98.

Email: nemes.laszlo@sph.unideb.hu

Phone: +36-52-411-600/55510

Sándor Kovács

Department of Economic Analysis and Statistics, Institute of Economic Analytical Methodology and Applied Informatics, University of Debrecen

Hungary, H-4032 Debrecen Böszörményi str. 138.

Email: kovacss@agr.unideb.hu

Phone: +36-30-378-41-12

\section{Antal Bugán}

Department of Behavioural Sciences, Medical and Health Science Center, University of Debrecen

Hungary, H-4032 Debrecen, Nagyerdei krt. 98.

Email: bugan.antal@sph.unideb.hu

Phone: +36-52- 411-600/ 56370 
Abstract

The purpose of this study was to determine health-related quality of life (HRQoL), fatigue, and posttraumatic growth (PTG) among patients with breast or prostate cancer during and following radiation therapy (RT). A total of 91 patients completed measures at three time points as follows: one or two weeks before the start of RT, in the fifth/sixth week of treatment, and four to six weeks post-treatment. Consistent with the previous literature, the results of this study revealed that RT was associated with declines in global HRQoL, Physical Well-being, Social/Family Well-being, and Functional Well-being, as well as an increase in fatigue. By four to six weeks post-treatment, these factors improved significantly and returned to baseline levels with the exception of Social/Family Well-being. The PTG total score and almost all of its subscale scores did not change significantly during the period of the study. However, the subscale of Spiritual Change for PTG significantly increased during the RT, and sustained this improvement at four to six weeks posttreatment. A significant negative correlation was found between Physical Well-being and PTG total score, whereas a significant positive correlation was revealed between Social/Family Wellbeing and PTG total score. The results from this analysis suggest that a significant decrease in HRQoL is evident in the fifth/sixth week of RT treatment. However, recovery of HRQoL occurs as quickly as a few weeks after the end of treatment. Moreover, patients with high ratings of Social/Family Well-being experience more positive psychological changes (PTG) resulting from the cancer experience.

Keywords: radiation therapy, health-related quality of life, fatigue, posttraumatic growth

\section{Introduction}

Recently, oncology and other divisions of medicine have placed great emphasis on measuring patients' health-related quality of life (HRQoL), which is an important indicator of treatment outcomes and provides information about the side effects of treatment (De Graeff et al. 1999; Müller et al. 2011; Wengström et al. 2000). Moreover, HRQoL is a relevant determinant of cancer patients' judgments and their adherence to oncological treatments (Frick et al. 2007). HRQoL is usually regarded as a multidimensional construct that includes several 
core domains, such as (1) mental functioning; (2) physical functioning; (3) role functioning; and (4) social functioning (Bowling 2001).

Radiotherapy (RT) is an increasingly used oncological treatment (Jones et al. 2011; Owen et al. 2006), with growing attention paid to its effects on HRQoL. Studies with RT recipients have measured HRQoL using the European Organization for Research and Treatment of Cancer Quality of Life Questionnaire (EORTC QOL-C30) (Dagnelie et al. 2007; Kenny et al. 2000; Lee et al. 2008; Stone et al. 2001), the Functional Assessment of Cancer Therapy Scale (FACT) (Deshields et al. 2005; Lee et al. 2001), and the Short Form-36 (SF-36) questionnaire (Madalinska et al. 2001; Stanton et al. 2001). These studies can be divided into the following two groups: half assess changes in HRQoL before, during, and after RT using a longitudinal design (e.g., Stone et al. 2001), whereas the other half compare the HRQoL of RT recipients to non-recipients (e.g., Madalinska et al. 2001) or RT recipients to healthy comparison subjects (e.g., Hann et al. 1998).

The number of longitudinal studies examining the quality of life of cancer patients during and after RT is, surprisingly, quite low. Several studies have shown that HRQoL significantly declines during RT, and then significantly improves some weeks after the treatment has ended. Deshields et al. (2005) found that breast cancer patients reported low levels of HRQoL at the conclusion of RT, but by two weeks post-treatment, their quality of life improved significantly and remained relatively stable at six months post-treatment. This finding suggests that psychological recovery occurs within a short time post-treatment. Stone et al. (2001) investigated HRQoL in a group of breast and prostate cancer patients receiving RT. They reported that there was a significant deterioration of global quality of life and some of its subscales (e.g., role functioning, cognitive 
functioning, and social functioning) between two points of measurement, which were pre-treatment (immediately prior to treatment) and within one week of the completion of RT. Another study found the same results with early-stage breast cancer patients (Jansen et al. 2000). Wengström et al. (2000) revealed a small decline in HRQoL during RT, but post-treatment scores seemed to equalize at follow-up measures (two weeks and three months after RT). A study by Dow and Lafferty (2000) demonstrated that overall quality of life and its subdomains (e.g., social, family, and psychological/spiritual functioning) declined from the start of RT to the midpoint of treatment, and then improved by six months after treatment ended (except for social functioning, which remained at a level that was lower than reported at the start of RT). By contrast, a number of studies have found that patients' quality of life remains unchanged during radiation treatment (Back et al. 2005; Lee et al. 2001; Lee et al. 2008). However, most of these studies did not measure HRQoL during RT, only before RT and after RT ended.

HRQoL declines during RT partly because RT may make existing symptoms worsen or may produce new physical symptoms (side effects) (Wengström et al. 2000). Fatigue is a common, highly disruptive complaint of patients undergoing external RT (Ahsberg and Fürst 2001), and its prevalence rates among patients receiving RT can reach $70 \%$ or more (Danjoux et al. 2007; Janaki et al. 2010; Jereczek-Fossa et al. 2002). A number of studies have demonstrated that fatigue increases during the course of RT, and then returns to pre-treatment levels several months after the completion of RT (Jansen et al. 2000; Lee et al. 2008; Stone et al. 2001). In a study by Wengström et al. (2000), fatigue worsened as the course of RT progressed, with a prevalence rate of over $85 \%$ among breast cancer patients in the third and fifth weeks of treatment $(87 \%$ and $90 \%$, respectively). The pre-treatment prevalence rate $(63 \%)$ returned by three 
months post-treatment. Fatigue has a relevant effect on patients' well-being, as it has been found to be the strongest determinant of overall quality of life (Dagnelie et al. 2007). Lee et al. (2008) revealed similar results by showing that fatigue is highly predictive of lower quality of life upon completion of RT and also at seven months after treatment ended. Moreover, fatigue is the most frequently mentioned symptom during RT (Wengström et al. 2000).

The diagnosis and treatment of cancer includes potentially psychologically traumatic experiences for patients. Individuals have to adapt to symptoms and side effects, which may cause meaningful disruptions across several life domains (Bellizzi et al. 2007; Mols et al. 2009). However, a growing body of recent literature has reported that a significant percentage of cancer patients (approximately 70-90\%) also experience positive life changes, namely posttraumatic growth (PTG) (Cordova et al. 2007; Lelorain et al. 2010; Schroevers and Teo 2008). The concept of PTG refers to positive changes that derive from struggling with highly stressful life events. These positive changes can be classified into the following five major categories: (1) a greater appreciation of life and a changed sense of priorities, (2) more meaningful relationships with others, (3) an increased sense of personal strength, (4) new possibilities for one's life, and (5) a richer existential and spiritual life (Tedeschi and Calhoun 2004).

In psycho-oncological research, HRQoL is sometimes positively related to PTG (Bower et al. 2005; Penedo et al. 2006; Urcuyo et al. 2005). For example, Lelorain et al. (2010) demonstrated that mental health and vitality subscales of overall quality of life were significantly positively related to PTG. Carver and Antoni (2004) reported in a longitudinal study that initial PTG predicted a higher quality of life five to eight years after diagnosis in breast cancer patients. In spite 
of these results, most studies have not revealed any significant associations between HRQoL and PTG (Dunn et al. 2011; Kinsinger et al. 2006; Park et al. 2010).

In the case of cancer, the traumatic stressor is complex because it may contain the diagnosis, severity and prognosis of cancer, as well as oncological treatments and their side effects (Sumalla et al. 2009). Some studies have tried to explore the nature of the relationship between the types of oncological treatments and PTG. In studies with cancer patients, receiving RT did not generally relate to PTG (Cordova et al. 2007; Mystakidou et al. 2008; Thombre et al. 2010). However, these cross-sectional studies measured PTG several months or years after the RT treatment, but not before, during, and after the RT. To date, no study in the scientific literature has explored changes in PTG before, during, and after the course of RT treatment.

The aim of the current longitudinal study was to investigate the patterns of change in HRQoL, fatigue, and PTG with a sample of breast and prostate cancer patients who were undergoing RT. First, we hypothesized that cancer patients' quality of life would significantly decrease during RT and that it would significantly improve after the treatment ended. Second, we hypothesized that PTG would not be related to HRQoL. Third, we assumed that the fatigue reported by patients would worsen during the course of RT and that it would decrease after the treatment ended. 


\section{Methods}

\section{Procedures}

The study sample was recruited from the Department of Radiotherapy at the University of Debrecen. The inclusion criteria for the study were the following: (1) no previous cancer history; (2) primary breast or prostate cancer without distant metastases; (3) imminent initiation of external beam radiotherapy; and (4) more than 18 years of age. Our sample consisted of patients with either breast or prostate cancer, given that these diseases have similar RT treatment regimens and cause similar impairments in the quality of life (Ransom et al. 2008). All questionnaires were administered three times. For the first wave, eligible patients were identified and then provided with information about the study by a psychologist after a session of radiation simulation (one or two weeks before the start of RT). Participants completed a written informed consent form and a questionnaire packet (T1). For the second and third waves, questionnaires were mailed to the patients in the fifth/sixth week of RT treatment (T2) and four to six weeks after the end of RT (T3). The study was approved by the Medical Ethical Committee from the Medical and Health Science Center at the University of Debrecen.

\section{Measures}

HRQoL. Quality of life was measured with the Hungarian translation (Webster et al. 2003) of the Functional Assessment of Cancer Therapy-General Questionnaire (FACT-G version 4; Cella et al. 1993), which was originally developed for and widely used with adult patients with any type of tumor. It consists of the following four subscales: Physical Well-being (PWB), Emotional 
Well-being (EWB), Social/Family Well-being (SFWB), and Functional Wellbeing (FWB). We explored feelings of fatigue with the following two individual items from the Physical Well-being subscale: "I have a lack of energy" and "I am forced to spend time in bed". FACT-G items were rated on a five-point Likert scale ranging from 0 (not at all) to 4 (very much). A total score was derived by summing the four subscale scores (range 0-108). Higher scores on the questionnaire indicate better HRQoL. For the present sample, the Cronbach's alphas for the FACT-G total score were 0.73 (T1), 0.69 (T2), and 0.80 (T3).

PTG. The Posttraumatic Growth Inventory (PTGI; Tedeschi and Calhoun 1996) is a 21-item self-report inventory of a person's perceptions of positive changes after a traumatic life event. The original English version assesses the following five domains of growth: Relating to Others, New Possibilities, Personal Strength, Spiritual Change, and Appreciation of Life. Items were rated on a sixpoint Likert scale ranging from 0 (I did not experience this change as a result of my cancer) to 5 (I experienced this change to a great degree as a result of my cancer). Adding up the scores of the five subscales yields a PTGI total score (range 0-105). The Hungarian version of the PTGI (Kulcsár et al. 2006) with the original 21 items and five subscales was used to measure cancer-related PTG. In this sample, the internal consistencies for the PTGI total scores were 0.81 (T1), $0.87(\mathrm{~T} 2)$, and $0.88(\mathrm{~T} 3)$.

Socio-demographic and medical variables. The following sociodemographic variables were assessed: age, gender, marital status, and education. The following medical variables were derived from patients' medical charts: the 
stage of the cancer, the type of treatment received (e.g., type of surgery, hormonal therapy, chemotherapy), and the length of time elapsed since diagnosis.

\section{Statistical analyses}

Descriptive statistics were measured for normalcy, and means, standard deviations, ranges, and counts for the variables were calculated. Chi-square tests, $t$-tests or Mann-Whitney $\mathrm{U}$ tests were used to determine whether those patients who did not complete all of the assessments differed from those who completed all of the assessments with regard to socio-demographic and medical variables. The relationships between the socio-demographic, medical variables and the PTG and FACT-G were determined using $t$-tests, the Mann-Whitney $U$ test, Welch's $t$ tests, analyses of variance (ANOVA), or the Kruskal-Wallis test. Pearson or Spearman correlations were calculated to reveal associations between the FACTG and PTG. Pairwise comparisons of the two individual fatigue-related items' scores at $\mathrm{T} 1, \mathrm{~T} 2$, and $\mathrm{T} 3$ were completed with Wilcoxon paired signed-rank tests. Finally, repeated measures analyses of variance were conducted to examine whether there were significant changes in FACT-G scores and PTGI scores throughout the duration of the study. Bonferroni's method was used for multiple comparisons of FACT-G scores and PTGI scores at T1, T2, and T3. The significance level for all analyses was defined as 0.05 . 


\section{Results}

\section{Participants}

A total of 152 individuals were approached to participate in this study before RT (T1) (84.2\% female patients; mean age: 59.1 years, $S D=10.7$, range 34 80). Of the 152 patients, $91(59.8 \%)$ completed questionnaires at $\mathrm{T} 2$ and $\mathrm{T} 3$ (84.6\% female patients; mean age: 58.7 years, $S D=10.6$, range 34-78). Attrition bias was examined by testing whether any systematic differences existed between patients missing at the follow-ups and patients who participated in all of the assessments. No significant differences were found between the two samples in socio-demographic and medical variables. Of the 91 patients, $65.9 \%$ were married or lived with a partner $(\mathrm{N}=60)$, and a significant number reported having a secondary education $(\mathrm{N}=60,65.9 \%)$. The mean length of time since diagnosis was 111.2 days ( $S D=77.5$, range 8-387). The disease stages at diagnosis were Stage I (46.1\%), Stage II (36.3\%), Stage III (14.3\%), or Stage IV (3.3\%). All patients had undergone some form of surgery, but only $15.4 \%(\mathrm{~N}=14)$ had undergone a mastectomy or prostatectomy. Many patients were treated by chemotherapy $(\mathrm{N}=43,47.3 \%)$ and a majority were treated by hormonal therapy $(\mathrm{N}=66,72.5 \%)$ (see Table 1).

\section{Relationships of socio-demographic and medical variables to HRQoL and PTG}

Preliminary analyses were conducted to examine whether sociodemographic or medical variables were related to HRQoL or PTG at the three measurement times. We found that neither PTGI total scores nor FACT-G total scores were significantly associated with any of the socio-demographic (i.e., age, 
gender, education, and marital status) or medical (i.e., stage, mastectomy/prostatectomy, hormonal therapy, chemotherapy, and time since diagnosis) variables at $\mathrm{T} 1, \mathrm{~T} 2$, and $\mathrm{T} 3$.

\section{Changes in HRQoL}

The means and standard deviations for the FACT-G total score and the FACT-G subscales are presented in Table 2. Overall $p$ values and $p$ values for the pairwise comparisons are also reported in Table 2. The FACT-G total scores significantly declined between $\mathrm{T} 1$ and $\mathrm{T} 2$, and then significantly increased between $\mathrm{T} 2$ and $\mathrm{T} 3$. No significant difference was found between the FACT-G total scores at $\mathrm{T} 1$ and $\mathrm{T} 3$. The same pattern was found for the PWB subscale: scores significantly decreased during RT (T2), and then returned to baseline levels four to six weeks after RT ended (T3). During RT (T2), the SFWB score was significantly lower than the baseline SFWB score (T1), and it remained low at the time of the third measurement (T3). EWB did not significantly change during the time period of the study. The FWB scores significantly decreased between T1 and $\mathrm{T} 2$, and then showed nonsignificant improvement by T3. Comparisons of the baseline HRQoL scores (T1) and the HRQoL scores at T3 revealed that none of the subscale scores at four to six weeks after RT ended (T3) were significantly different from the baseline scores (T1), except for the SFWB score. This result means that all of the well-being scores returned to the baseline scores four to six weeks after RT. 


\section{Changes in PTG}

The means and standard deviations for the PTGI total score and the PTGI subscales are presented in Table 2. Overall $p$ values and $p$ values for the pairwise comparisons are also reported in Table 2. The PTGI total score and the subscales for New Possibilities, Personal Strength, and Relating to Others remained relatively stable throughout the measurements. The Appreciation of Life scores increased but not significantly at $\mathrm{T} 2$ compared with $\mathrm{T} 1$, and then significantly declined between T2 and T3. The Spiritual Change scores significantly increased during RT (T2), and this improvement was maintained four to six weeks after RT ended (T3).

\section{Relationships between HRQoL and PTG}

There were no statistically significant correlations between the PTGI total scores and the FACT-G total scores (at T1, T2, and T3) (see Table 3). However, significant negative correlations were found between PWB scores and PTGI total scores. The strongest correlation was between the PWB score at T1 and the PTGI total score at T3 $(r=-0.26, p<0.01)$. Moreover, significant positive correlations were revealed between SFWB subscales and PTGI total scores at all points of measurement. The strongest correlation was between the SFWB subscale and the PTGI total score at T1 $(r=0.34 ; p<0.01)$.

\section{Changes in fatigue}

Changes in feelings of fatigue were calculated by separating the two fatiguerelated items from the PWB subscale of the FACT-G (see Table 4). The results derived from these two items show the same pattern of change, in that patients felt 
significantly more fatigue during RT (T2) compared with the baseline measurement (T1). However, fatigue significantly decreased by four to six weeks after RT ended (T3). Thus, the feeling of fatigue increased during RT, but it returned to the baseline level some weeks after the treatment ended.

\section{Discussion}

This study is the only one available in the psycho-oncological literature that examined the relationship between HRQoL and PTG among breast and prostate cancer patients undergoing RT. We used a longitudinal design to measure HRQoL and PTG before, during (in the fifth/sixth week of the treatment), and after RT (four to six weeks after the end of treatment).

According to our results, neither PTGI total scores nor FACT-G total scores were significantly associated with any of the socio-demographic or medical variables during the study period. We found a significant deterioration in overall quality of life (FACT-G total) and PWB at the fifth/sixth week of RT. However, by four to six weeks after RT had ended, both of these variables showed improvement and had returned to their baseline levels. The FWB score decreased during RT, and then started to increase by four to six weeks post-treatment, but only to a small degree.

EWB was the only domain of HRQoL that remained unchanged, which is in line with results from a previous study reporting that RT recipients and healthy comparison subjects do not differ with regard to levels of anxiety and depressive symptoms (Hann et al. 1998). This result may suggest that a relative emotional equilibrium was typical for these patients during and shortly after RT in contrast with the upheaval caused by the diagnosis. 
We observed a significant decline in the SFWB score during RT and that this was the only domain of HRQoL that did not return to the baseline level after RT ended. This result is in line with the study of Dow and Lafferty (2000) that found that social functioning significantly decreased during RT and remained lower by six months post-treatment. Although the specific cause of this decrease in SFWB could not be determined in this study, it is possible that during and shortly after RT patients temporarily withdraw from their social support system because of the experienced side effects caused by the RT.

Overall, we found that all of the well-being scores (except for EWB) significantly decreased during RT. However, by four to six weeks after RT had ended, all of them improved and returned to their baseline scores (except for SFWB, which did not return). The changes in HRQoL within this sample were similar to those reported in previous studies (Deshields et al. 2005; Wengström et al. 2000). For example, Stone et al. (2001) observed significant decreases in role, cognitive, and social functioning by the last week of RT. They also found that emotional functioning did not change during this period. A study conducted by Dow and Lafferty (2000) revealed that, by the midpoint of RT, significant deterioration had occurred in social, family, and psychological/spiritual functioning. By six months post-treatment, all of these factors improved with the exception of social functioning, which remained low.

Our hypothesis that fatigue reported by patients may worsen during the course of RT was supported by the results of this study. A clinically significant increase in fatigue was found during the RT, but it returned to the baseline level four to six weeks after the end of treatment. This finding is consistent with some previous studies that found the same pattern of change in fatigue among cancer patients receiving RT (Jansen et al. 2000; Lee et al. 2008; Stone et al. 2001). 
The overall PTG and most of its subscales did not change significantly during the time period of this study. Only two subscales were exceptions: Appreciation of Life and Spiritual Change. Appreciation of Life showed an increasing trend during RT, and then it significantly declined four to six weeks after the treatment ended. It is possible that receiving RT treatment constantly reminds a person of his/her illness and the possible danger of death, which can enhance feelings of appreciation and respect for life. However, after the treatment this "memento" may disappear, making the Appreciation of Life decrease. Moreover, Spiritual Change significantly increased during RT, and this improvement was maintained four to six weeks post-treatment. RT treatment may serve as a reminder of the threat of death, which can precipitate sensitivity to the transcendent. This experience may continue after the RT, as the signs of recovery (e.g., better HRQoL) confirm the relationship with the transcendent (e.g., God).

As expected, no significant associations were found between the PTGI total scores and the FACT-G total scores. Nevertheless, we found significant negative correlations between PWB and PTGI total scores: people with lower PWB reported greater PTG. Physical symptoms may remind patients of the threat caused by their illness and, as other studies have shown, greater feelings of threat may prompt more positive changes (PTG) (Barakat et al. 2006; Lechner et al. 2003). In addition, positive correlations were reported between SFWB and PTGI total scores, indicating that patients with greater perceived social support experienced more positive changes. Several studies have found that social support is a determining factor in finding benefits in a life-threatening illness (Bellizzi et al. 2007; Dunn et al. 2011; Schroevers et al. 2010). The possibility for patients to disclose negative cancer-related emotions (e.g., fear) and the empathy and 
compassion coming from family members and friends can contribute to greater awareness of positive changes that result from an illness.

Although we found significant impairment in HRQoL during RT, it is important to note that RT has a highly relevant role in the curative and palliative treatment of cancer. For example, RT can significantly decrease the incidence of intra-breast tumor reappearance in the case of breast cancer (Veronesi et al. 2001). Moreover, this decline in quality of life is only transient, as Deshiels et al. (2005) demonstrated that psychological "recovery" occurs two weeks after the end of RT. It may be important for future research to compare changes in HRQoL during chemotherapy to changes in HRQoL during RT, since as Conner-Spady et al. (2005) found, the pattern of change in HRQoL during chemotherapy can be similar to our results.

The present study has several limitations. First, the sample consisted primarily of female breast cancer patients (partly because prostate cancer patients were more likely to refuse study participation). It limits the generalizability of the results to other cancer populations. Second, we measured fatigue with two individual items of the PWB subscale. A questionnaire that specifically assesses fatigue may be more useful and valid for exploring this relevant side effect of RT. Third, the time interval for this study was relatively short. A longitudinal study that lasts several years could explore both the short-term and long-term side effects of RT. Fourth, our study did not make a baseline assessment of HRQoL and PTG at the time of diagnosis, which would have provided a fuller picture of the changes in HRQoL and PTG. Fifth, HRQoL does not provide information about all of the effects of treatment that study participants experience during RT. Therefore, the application of a radiotherapy-specific instrument which measures the side effects of RT would be highly recommended. 
The results of our study have important clinical implications. Patients with breast and prostate cancers should be informed about the probable side effects of RT and their effective management before the start of treatment. For example, exercise (e.g., walking) is a highly recommended and useful technique for alleviating fatigue and improving physical functioning during and after RT (Hwang et al. 2008; Mock et al. 2001). In addition, given that SFWB was the only domain of HRQoL that remained low after treatment, health care professionals should emphasize promotion of the social well-being of patients undergoing RT. This can be realized by either organizing support groups (Lazányi et al. 2010) or providing personal counseling, which could be helpful to avoid or decrease the social withdrawal of RT recipients. Our results show that only those patients who feel higher SFWB are able to recognize the psychological benefits (PTG) resulting from the cancer. 
Table 1 Socio-demographic and medical characteristics of the sample (N=91)

\begin{tabular}{|c|c|c|c|c|c|c|c|c|c|}
\hline \multirow[b]{2}{*}{ Variable } & \multicolumn{3}{|c|}{ Female } & \multicolumn{3}{|c|}{ Male } & \multicolumn{3}{|c|}{ Total } \\
\hline & $M$ & $S D$ & $\%$ & $M$ & $S D$ & $\%$ & $M$ & $S D$ & $\%$ \\
\hline Age in years ${ }^{\mathrm{a}}$ & 57.1 & 10.5 & & 67.6 & 6.1 & & 58.7 & 10.6 & \\
\hline \multicolumn{10}{|l|}{ Gender } \\
\hline Female & & & & & & & & & 84.6 \\
\hline Male & & & & & & & & & 15.4 \\
\hline \multicolumn{10}{|l|}{ Marital status } \\
\hline \multicolumn{10}{|l|}{ Married or live with a } \\
\hline partner & & & 64.9 & & & 71.4 & & & 65.9 \\
\hline Not married ${ }^{\mathrm{b}}$ & & & 35.1 & & & 28.6 & & & 34.1 \\
\hline \multicolumn{10}{|l|}{ Educational level $^{c}$} \\
\hline Primary education or less & & & 23.4 & & & 28.6 & & & 24.2 \\
\hline Secondary education & & & 66.2 & & & 64.3 & & & 65.9 \\
\hline Higher education & & & 10.4 & & & 7.1 & & & 9.9 \\
\hline \multicolumn{10}{|l|}{ Cancer site } \\
\hline Breast & & & & & & & & & 84.6 \\
\hline Prostate & & & & & & & & & 15.4 \\
\hline \multicolumn{10}{|l|}{ Stage } \\
\hline$I^{d}$ & & & 54.6 & & & 0 & & & 46.1 \\
\hline II $^{\mathrm{d}}$ & & & 32.4 & & & 57.2 & & & 36.3 \\
\hline III $^{\mathrm{d}}$ & & & 13 & & & 21.4 & & & 14.3 \\
\hline IV & & & 0 & & & 21.4 & & & 3.3 \\
\hline Time since diagnosis (days) & 106.2 & 77.8 & & 138.7 & 72.7 & & 111.2 & 77.5 & \\
\hline \multicolumn{10}{|l|}{ Type of treatment } \\
\hline$(Y e s)^{\mathrm{e}}$ & & & 14.2 & & & 21.4 & & & 15.4 \\
\hline Hormonal therapy (Yes) ${ }^{\mathrm{d}}$ & & & 68.8 & & & 92.8 & & & 72.5 \\
\hline Chemotherapy (Yes) ${ }^{\mathrm{d}}$ & & & 54.5 & & & 7.1 & & & 47.3 \\
\hline
\end{tabular}


Note:

${ }^{\mathrm{a}}$ Genders differ at $p<0.01$.

${ }^{\mathrm{b}}$ Includes single, divorced, and widowed patients.

${ }^{\mathrm{c}}$ Primary education: primary school; Secondary education: secondary school, trade school, technical college, grammar school; Higher education: college, university.

${ }^{\mathrm{d}}$ Genders differ at $p<0.001$.

${ }^{\mathrm{e}}$ Mastectomy: among patients with breast cancer; Prostatectomy: among patients with prostate cancer. 
Table 2 Repeated measures analysis of variance of quality of life and posttraumatic growth

\begin{tabular}{|c|c|c|c|c|c|c|c|}
\hline & \multicolumn{3}{|c|}{ Mean $(S D)$} & \multicolumn{4}{|c|}{$p^{*}$} \\
\hline & $\mathrm{T} 1$ & $\mathrm{~T} 2$ & $\mathrm{~T} 3$ & Overall & T1 vs. T2 & $\mathrm{T} 2$ vs. T3 & T1 vs. T3 \\
\hline FACT-G & 81.9 (17.2) & $75.4(17.6)$ & $79.4(17.8)$ & $0.001^{\dagger}$ & $0.000^{\dagger}$ & $0.001^{\dagger}$ & 0.232 \\
\hline PWB & $20.9(6.9)$ & $19.1(6.6)$ & $21.2(6.6)$ & $0.001^{\dagger}$ & $0.018^{\dagger}$ & $0.000^{\dagger}$ & 1.000 \\
\hline SFWB & $23.7(4.7)$ & $21.4(5.5)$ & $21.6(5)$ & $0.001^{\dagger}$ & $0.001^{\dagger}$ & 1.000 & $0.001^{\dagger}$ \\
\hline EWB & $17.4(5.2)$ & $17.1(5.2)$ & $17.6(5.5)$ & 0.985 & 1.000 & 0.369 & 1.000 \\
\hline FWB & $19.8(6.4)$ & $17.7(6.6)$ & $18.8(6.1)$ & $0.002^{\dagger}$ & $0.004^{\dagger}$ & 0.116 & 0.315 \\
\hline PTGI & $63(23.3)$ & $67.5(22.3)$ & $64.9(23.9)$ & 0.067 & 0.134 & 0.222 & 0.945 \\
\hline $\mathrm{AL}$ & $9.9(4.1)$ & $10.8(4.0)$ & $10(4.3)$ & $0.027^{\dagger}$ & 0.089 & $0.019^{\dagger}$ & 1.000 \\
\hline NP & $12.3(7.7)$ & $13.9(6.8)$ & $13.4(6.9)$ & 0.051 & 0.115 & 0.819 & 0.284 \\
\hline PS & $13.3(4.7)$ & $13.6(4.5)$ & $13.5(4.8)$ & 0.774 & 1.000 & 1.000 & 1.000 \\
\hline $\mathrm{SC}$ & $3.3(3.2)$ & $4.3(3.1)$ & $4.1(3.1)$ & $0.003^{\dagger}$ & $0.006^{\dagger}$ & 0.850 & $0.005^{\dagger}$ \\
\hline $\mathrm{RO}$ & $24(8.9)$ & $24.6(7.4)$ & $23.7(8.1)$ & 0.437 & 1.000 & 0.370 & 1.000 \\
\hline
\end{tabular}

Abbreviations: $\mathrm{SD}=$ standard deviation; T1=Time 1 (before radiotherapy); T2=Time 2 (the fifth/sixth week of radiotherapy); T3=Time 3 (four to six weeks after radiotherapy); FACT-G=Functional Assessment of Cancer Therapy-General total score; PWB=Physical Well-being; SFWB=Social/ Family Well-being; EWB=Emotional Well-being; FWB=Functional Well-being; PTGI=Posttraumatic Growth Inventory total score; AL=Appreciation of Life; NP=New Possibilities; $\mathrm{PS}=$ Personal Strength; $\mathrm{SC}=$ Spiritual Change; $\mathrm{RO}=$ Relating to Others.

${ }^{*} \mathrm{P}$ values of repeated measures analyses of variance and Bonferroni's multiple comparisons.

${ }^{\dagger}$ Statistically significant. 
Table 3 Intercorrelations between health-related quality of life and posttraumatic growth at Time 1, Time 2, and Time 3

\begin{tabular}{lccc}
\hline $\begin{array}{l}\text { HRQoL } \\
\text { domains }\end{array}$ & PTGI (T1) & PTGI (T2) & PTGI (T3) \\
\hline FACT-G (T1) & 0.10 & -0.08 & -0.05 \\
FACT-G (T2) & 0.01 & 0.05 & 0.00 \\
FACT-G (T3) & -0.01 & 0.04 & 0.00 \\
PWB (T1) & -0.16 & $-0.22^{*}$ & $-0.26^{* *}$ \\
PWB (T2) & -0.16 & -0.09 & -0.18 \\
PWB (T3) & -0.17 & -0.19 & $-0.21^{*}$ \\
SFWB (T1) & $0.34^{* *}$ & 0.05 & 0.11 \\
SFWB (T2) & 0.28 & $0.26^{*}$ & $0.2 *^{*} *$ \\
SFWB (T3) & 0.35 & $0.27^{*}$ & $0.27^{* *}$ \\
EWB (T1) & -0.00 & -0.06 & -0.08 \\
EWB (T2) & -0.04 & -0.14 & -0.09 \\
EWB (T3) & 0.09 & 0.08 & -0.10 \\
FWB (T1) & 0.14 & 0.00 & 0.09 \\
FWB (T2) & 0.02 & 0.14 & 0.05 \\
FWB (T3) & -0.03 & 0.16 & 0.12 \\
\hline
\end{tabular}

Abbreviations: HRQoL=health-related quality of life; PTGI=Posttraumatic Growth Inventory total score; T1=Time 1 (before radiotherapy); T2=Time 2 (the fifth/sixth week of radiotherapy); T3=Time 3 (four to six weeks after radiotherapy); FACT-G=Functional Assessment of Cancer Therapy-General total score; PWB=Physical Well-being; SFWB=Social/Family Well-being; EWB=Emotional Well-being; FWB=Functional Wellbeing.

${ }^{*} p<0.05 ; * * p<0.01$. 
Table 4 Changes in fatigue

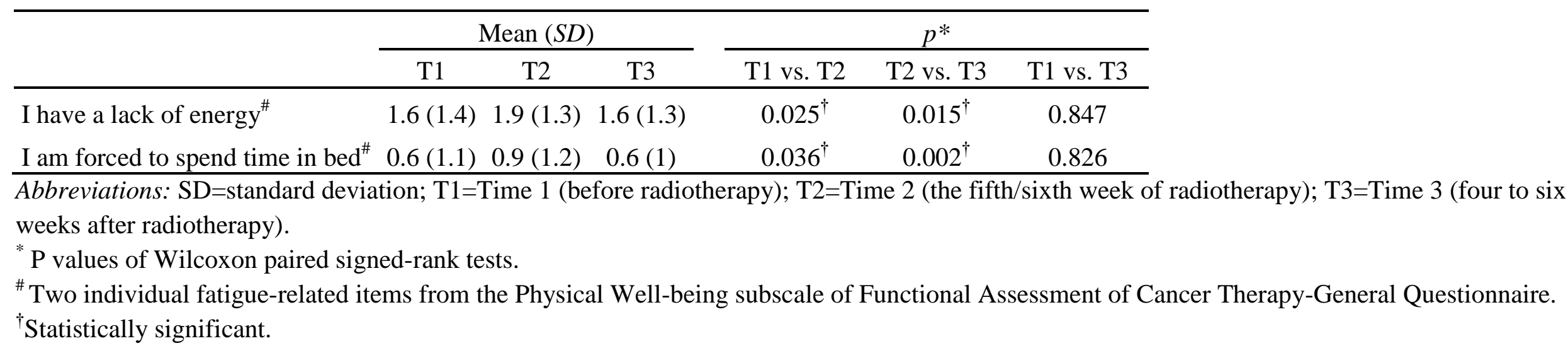




\section{References}

Ahsberg, E., \& Fürst, C. J. (2001). Dimensions of fatigue during radiotherapy. An application of the Swedish Occupational Fatigue Inventory (SOFI) on cancer patients. Acta Oncologica, 40(1), 37-43.

Back, M., Ahern, V., Delaney, G., Graham, P., Steigler, A., \& Wratten, C. (2005). Absence of adverse early quality of life outcomes of radiation therapy in breast conservation therapy for early breast cancer. Australasian Radiology, 49, 39-43.

Barakat, L. P., Alderfer, M. A., \& Kazak, A. E. (2006). Posttraumatic growth in adolescent survivors of cancer and their mothers and fathers. Journal of Pediatric Psychology, 31(4), 413-419.

Bellizzi, K. M., Miller, M. F., Arora, N. K., \& Rowland, J. H. (2007). Positive and negative life changes experienced by survivors of Non-Hodgkin's lymphoma. Annals of Behavioral Medicine, 34(2), 188-199. Bower, J. E., Meyerowitz, B. E., Desmond, K. A., Bernaards, C. A., Rowland, J. H., \& Ganz, P. A. (2005). Perceptions of positive meaning and vulnerability following breast cancer: Predictors and outcomes among longterm breast cancer survivors. Annals of Behavioral Medicine, 29(3), 236-245.

Bowling, A. (2001). Measuring disease: A review of disease specific quality of life measurement scales. Philadelphia: Open University Press.

Carver, C. S., \& Antoni, M. H. (2004). Finding benefit in breast cancer during the year after diagnosis predicts better adjustment 5 to 8 years after diagnosis. Health Psychology, 23(6), 595-598.

Cella, D. F., Tulsky, D. S., Gray, G., Sarafian, B., Linn, E., Bonomi, A., et al. (1993). The Functional Assessment of Cancer Therapy scale: Development and validation of the general measure. Journal of Clinical Oncology, 11(3), 570-579.

Cordova, M. J., Giese-Davis, J., Golant, M., Kronenwetter, C., Chang, V., \& Speigel, D. (2007). Breast cancer as trauma: Posttraumatic stress and posttraumatic growth. Journal of Clinical Psychology in Medical Settings, 14, 308-319.

Dagnelie, P. C., Pijls-Johannesma, M. C. G., Lambin, P., Beijer, S., De Ruyssher, D., \& Kempen, G. I. J. M. (2007). Impact of fatigue on overall quality of life in lung and breast cancer patients selected for high-dose radiotherapy. Annals of Oncology, 18, 940-944.

Danjoux, C., Gardner, S., \& Fitch, M. (2007). Prospective evaluation of fatigue during a course of curative radiotherapy for localised prostate cancer. Support Care Cancer, 15, 1169-1176.

De Graeff, A., de Leeuw, J. R. J., Ros, W. J. G., Hordijk, G. J., Blijham, G. H., \& Winnubst, J. A. M. (1999). A prospective study on quality of life of patients with cancer of the oral cavity or oropharynx treated with surgery with or without radiotherapy. Oral Oncology, 35, 27-32.

Deshield, T., Tibbs, T., Fan, M., Bayer, L., Taylor, M., \& Fisher, E. (2005). Ending treatment: The course of emotional adjustment and quality of life among breast cancer survivors immediately following radiation therapy. Support Care Cancer, 13, 1018-1026.

Dow, K. H., \& Lafferty, P. (2000). Quality of life, survivorship, and psychosocial adjustment of young women with breast cancer after breast-conserving surgery and radiation therapy. Oncology Nursing Forum, 27(10), 1555-1564. 
Dunn, J., Occhipinti, S., Campbell, A., Ferguson, M., \& Chambers, S. K. (2011). Benefit finding after cancer. The role of optimism, intrusive thinking and social environment. Journal of Health Psychology, 16(1), 169-177. Frick, E., Tyroller, M., \& Panzer, M. (2007). Anxiety, depression and quality of life of cancer patients undergoing radiation therapy: a cross-sectional study in a community hospital outpatient centre. European Journal of Cancer Care, 16, 130-136.

Hann, D. M., Jacobsen, P., Martin, S., Azzarello, L., \& Greenberg, H. (1998). Fatigue and quality of life following radiotherapy for breast cancer: A comparative study. Journal of Clinical Psychology in Medical Settings, 5(1), 19-33.

Hwang, J. H., Chang, H. J., Shim, Y. H., Park, W. H., Park, W., Huh, S. J., et al. (2008). Effects of supervised exercise therapy in patients receiving radiotherapy for breast cancer. Yonsei Medical Journal, 49(3), 443-450. Janaki, M. G., Kadam, A. R., Mukesh, S., Nirmala, S., Ponni, A., Ramesh, B. S., et al. (2010). Magnitude of fatigue in cancer patients receiving radiotherapy and its short term effect on quality of life. Journal of Cancer Research and Therapeutics, 6(1), 22-26.

Jansen, S. J. T., Stiggelbout, A. M., Nooij, M. A., Noordijk, E. M., \& Kievit, J. (2000). Response shift on quality of life measurement in early-stage breast cancer patients undergoing radiotherapy. Quality of Life Research, 9 , 603-615.

Jereczek-Fossa, B. A., Marsiglia, H. R., \& Orecchia, R. (2002). Radiotherapy-related fatigue. Critical Reviews in Oncology/Hematology, 41, 317-325.

Jones, C. U., Hunt, D., McGowan, D. G., Amin, M. B., Chetner, M. P., Bruner, D. W., et al. (2011).

Radiotherapy and short-term androgen deprivation for localized prostate cancer. The New England Journal of Medicine, 365(2), 107-118.

Kenny, P., King, L. M., Shiell, A., Seymour, J., Hall, J., Langlands, A., et al. (2000). Early stage breast cancer: Costs and quality of life one year after treatment by mastectomy or conservative surgery and radiation therapy. The Breast, 9, 37-44.

Kinsinger, D. P., Penedo, F. J., Antoni, M. H., Dahn, J. R., Lechner, S., \& Schneiderman, N. (2006).

Psychosocial and sociodemographic correlates of benefit-finding in men treated for localized prostate cancer. Psycho-Oncology, 15, 954-961.

Kulcsár, Zs., Rózsa, S., \& Reinhardt, M. (2006). A poszttraumás növekedés feltételei és empirikus vizsgálata [Conditions and empirical examination of posttraumatic growth]. Budapest: Trefort.

Lazányi, K. R., Molnár, P., Bugán, A., Damjanovich, L., Garami, Z., Fülöp, B., et al. (2010). The 'HÍVÁS’ Club: Social support in post cancer recovery. Clinical and Experimental Medical Journal, 4(1), 105-113.

Lechner, S. C., Zakowski, S. G., Antoni, M. H., Greenhawt, M., Block, K., \& Block, P. (2003). Do sociodemographic and disease-related variables influence benefit-finding in cancer patients? Psycho-Oncology, $12,491-499$.

Lee, W. R., Hall, M. C., McQellon, R. P., Case, L. D., \& McCullough, D. L. (2001). A prospective quality-oflife study in men with clinically localized prostate carcinoma treated with radical prostatectomy, external beam radiotherapy, or interstitial brachytherapy. International Journal of Radiation Oncology, Biology, Physics, 51(3), 614-623.

Lee, T. S., Kilbreath, S. L., Refshauge, K. M., Pendlebury, S. C., Beith, J. M., \& Lee, M. J. (2008). Quality of life of women treated with radiotherapy for breast cancer. Support Care Cancer, 16, 399-405. 
Lelorain, S., Bonnaud-Antignac, A., \& Florin, A. (2010). Long term posttraumatic growth after breast cancer: Prevalence, predictors and relationships with psychological health. Journal of Clinical Psychology in Medical Settings, 17, 14-22.

Madalinska, J. B., Essink-Bot, M., de Koning, H. J., Kirkels, W. J., van der Maas, P. J., \& Schröder, F. H. (2001). Health-related quality-of-life effects of radical prostatectomy and primary radiotherapy for screendetected or clinically diagnosed localized prostate cancer. Journal of Clinical Oncology, 19, 1619-1628. Mock, V., Pickett, M., Ropka, M. E., Muscari, E., Stewart, K. J., Rhodes, V. A., et al. (2001). Fatigue and quality of life outcomes of exercise during cancer treatment. Cancer Practice, 9(3), 119-127.

Mols, F., Vingerhoets, A. J. J. M., Coebergh, J. W. W., \& van de Poll-Franse, L. V. (2009). Well-being, posttraumatic growth and benefit finding in long-term breast cancer survivors. Psychology and Health, 24(5), 583-595.

Müller, A., Kerényi, E., \& Könyves, E. (2011). Effects of climate therapy and rehabilitation in Mátra Medical Institute. Applied Studies in Agribusiness and Commerce, 3-4, 39-42.

Mystakidou, K., Tsilika, E., Parpa, E., Kyriakopulos, D., Malamos, N., \& Damigos, D. (2008). Personal growth and psychological distress in advanced breast cancer. The Breast, 17, 382-386.

Owen, R. J., Ashton, A., Bliss, J. M., Homewood, J., Harper, C., Hanson, J., et al. (2006). Effect of radiotherapy fraction size on tumour control in patients with early-stage breast cancer after local tumour excision: Long-term results of a randomised trial. The Lancet Oncology, 7, 467-471.

Park, C. L., Chmielewski, J., \& Blank, T. O. (2010). Post-traumatic growth: Finding positive meaning in cancer survivorship moderates the impact of intrusive thoughts on adjustment in younger adults. Psycho-Oncology, 19, 1139-1147.

Penedo, F. J., Molton, I., Dahn, J. R., Shen, B., Kinsinger, D., Traeger, L., et al. (2006). A randomized clinical trial of group-based cognitive-behavioral stress management in localized prostate cancer: Development of stress management skills improves quality of life and benefit finding. Annals of Behavioral Medicine, 31(3), 261-270. Ransom, S., Sheldon, K. M., \& Jacobsen, P. B. (2008). Actual change and inaccurate recall contribute to posttraumatic growth following radiotherapy. Journal of Consulting and Clinical Psychology, 76(5), 811-819.

Schroevers, M. J., Helgeson, V. S., Sanderman, R., \& Ranchor, A. V. (2010). Type of social support matters for prediction of posttraumatic growth among cancer survivors. Psycho-Oncology, 19, 46-53.

Schroevers, M. J., \& Teo, I. (2008). The report of posttraumatic growth in Malaysian cancer patients: Relationships with psychological distress and coping strategies. Psycho-Oncology, 17, 1239- 1246.

Stanton, A. L., Krishnan, L., \& Collins, C. A. (2001). Form or function? Part 1. Subjective cosmetic and functional correlates of quality of life in women treated with breast-conserving surgical procedures and radiotherapy. Cancer, 91, 2273-2281.

Stone, P., Richards, M., A'Hern, R., \& Hardy, J. (2001). Fatigue in patients with cancers of the breast or prostate undergoing radical radiotherapy. Journal of Pain and Symptom Management, 22(6), 1007-1015.

Sumalla, E. C., Ochoa, C. \& Blanco, I. (2009). Posttraumatic growth in cancer: Reality or illusion? Clinical Psychology Review, 29, 24-33.

Tedeschi, R. G., \& Calhoun, L. G. (1996). The Posttraumatic Growth Inventory: Measuring the positive legacy of trauma. Journal of Traumatic Stress, 9(3), 455-471.

Tedeschi, R. G., \& Calhoun, L. G. (2004). Posttraumatic growth: Conceptual foundations and empirical evidence. Psychological Inquiry, 15(1), 1-18. 
Thombre, A., Sherman, A. C., \& Simonton, S. (2010). Posttraumatic growth among cancer patients in India. Journal of Behavioral Medicine, 33, 15-23.

Urcuyo, K. R., Boyers, A. E., Carver, C. S., \& Antoni, M. H. (2005). Finding benefit in breast cancer: Relations with personality, coping, and concurrent well-being. Psychology and Health, 20(2), 175-192.

Veronesi, U., Marubini, E., Mariani, L., Galimberti, V., Luini, A., Veronesi, P., et al. (2001). Radiotherapy after breast-conserving surgery in small breast carcinoma: Long-term results of a randomized trial. Annals of Oncology, 12, 997-1003.

Webster, K., Cella, D., \& Yost, K. (2003). The Functional Assessment of Chronic Illness Therapy (FACIT) Measurement System: Properties, applications, and interpretation. Health and Quality of Life Outcomes, 1(79), $1-7$.

Wengström, Y., Häggmark, C., Strander, H., \& Forsberg, C. (2000). Perceived symptoms and quality of life in women with breast cancer receiving radiation therapy. European Journal of Oncology Nursing, 4(2), 78-88. 